\title{
Frequency and Type of Medication Discrepancies in One Tertiary Care Hospital
}

$\overline{\text { Jennifer Turple, Neil J. MacKinnon and Bryan Davis }}$

\begin{abstract}
Background/Objective: Discrepancies in records used within the medication use system have been identified as a contributing factor of medication errors. The objective of this study was to determine the frequency and type of discrepancies in the medication use system in one tertiary care hospital.

Methods: Using a sample of patients (convenience sampling technique), the physician's orders, the nursing medication administration record and the pharmacy profile were compared in an attempt to identify discrepancies among them. A discrepancy was defined as a deviation from the physician's order as written in the chart. Each discrepancy was categorized according to seven components of the medication order, its location in the medication use process and its mode of delivery.

Results: One thousand, four hundred twenty-four orders representing 197 patients from 13 nursing units were sampled for this study. Thirteen percent of the orders were discrepant and $61 \%$ of patients had at least one discrepancy. The most frequent types of discrepancies were drug omissions and unordered drugs.

Discussion: The discrepancies identified in this study suggest that either orders are not reaching pharmacy or orders are not being processed appropriately in pharmacy. The location of discrepancies also suggests that there are deficiencies in communication between healthcare professionals.
\end{abstract}

\section{Background/Objective}

The safety of the medication use system (MUS) is an issue which is a concern in many healthcare organizations today. This problem was clearly identified in the Institute of Medicine's report To Err Is Human (Kohn et al. 1999). The Canadian Adverse Events Study (Baker et al. 2004) helped to quantify the magnitude of this problem in the Canadian inpatient environment. In that study, almost one-quarter of all the adverse events identified were drug- or fluid-related. The annual cost of preventable drug-related morbidity and mortality in Canada has been estimated to be $\$ 11$ billion per year in older adults alone (Kidney and MacKinnon 2001).

Problems related to documentation and communication in the MUS are commonly cited in studies. Of 134 patients in the intervention arm of a study (Nickerson et al. 2005) at the Moncton Hospital, NB, 96.3\% (129) patients had at least one drug-therapy problem for monitoring, while $39.6 \%$ (53) patients had a drug-therapy inconsistency or omission. All of these problems were identified just prior to discharge from hospital. In another study that focused on unintended discrepancies on admission, 53.6\% of patients had at least one such discrepancy (Cornish et al. 2005). Current efforts by the Canadian Council on Health Services Accreditation (2006) and the Safer Healthcare Now! (2006) campaign directed toward 
the documentation and communication of the patient's prescription profile through medication reconciliation will hopefully improve the situation.

Given the importance of identifying potential flaws in the MUS, the goal of this study was to identify discrepancies in the documentation throughout the MUS in the Queen Elizabeth II Health Sciences Centre (QEII HSC) in Halifax, and to assess whether these discrepancies could be contributing to medication error.

\section{Methods}

This study was approved by the Capital Health Research Ethics Board. The MUS at the QEII HSC relies heavily on three documents, which are independently maintained by physicians, nurses and pharmacists. These documents include: (1) the physician's handwritten order located in the patient's chart, (2) the medication administration record (MAR), which is transcribed by nurses and guides subsequent medication administration and (3) the pharmacistmaintained electronic medication profile, which guides the dispensing of medications. In this study, these key documents were compared to identify and evaluate discrepancies. A discrepancy was defined as a deviation (spelling excluded) from the physician's order as it was written in the chart.

A sample of patients was selected using a convenience sampling technique. These patients were admitted to acute care beds on a nursing unit that utilized the unit-dose system at the Halifax Infirmary Site of the QEII HSC between April and May 2003. Patients were excluded if no scheduled medications were ordered.

For each patient selected, photocopies of all physician's orders, the current "scheduled" MAR (as opposed to the "prn" MAR), and the current (RxTFC TM [BDM Information Systems Ltd., Saskatoon]) electronic pharmacy profile were obtained. For simplicity, the following orders were excluded from analysis: (1) newly written orders that had not been processed throughout the entire MUS (i.e., transcribed and dispensed), (2) "as needed" orders and (3) "one time only" orders.

At the end of the two-month data collection period, the orders were reviewed to identify all medications the patient was to be actively receiving at the time of document collection. Once all active orders had been identified, each one was crossreferenced to the other MUS documentation (see Figure 1).

Discrepancies between the original physician's order from the chart, the nurse's transcription in the MAR and the pharmacist's order entry in RxTFC were noted. Specifically, seven components of the order were examined for discrepancies. These components (or types) included: dosage, route of administration, frequency or duration, unordered drugs, omission of drugs, dosage form and improper administration. Each discrepancy was then categorized according to the type and the location of the discrepancy (e.g., between the physician's order and pharmacy profile). For each discrepancy, the drug involved was categorized according to the mode of delivery (e.g., unit dose, floor stock) to the patient.

A panel of two pharmacists and two nurses, who work within the MUS at the QEII HSC, assisted in assessment of discrepancies. The investigator prepared the documents for review, which included the discrepant transcription and/or pharmacy entry, the category of discrepancy and the delivery method. Using their professional judgment, each panel member independently assessed the likelihood that each discrepancy, as described, led to an actual error in drug administration. The likelihood was documented using a 3-point Likert scale ( $1=$ Very Unlikely, $2=$ Possible and $3=$ Very Likely).

Statistical analysis was completed using SPlus 6.0 software. Descriptive statistics were used to qualify patient characteristics, discrepancy characteristics and rates. Linear regression and proportion testing were also used in the data analysis.

\section{Results}

The previously specified documentation for 197 patients was included in the study over the two-month data collection period. There were 100 (51\%) male and 97 (49\%) female patients, with an average age $( \pm S D)$ of $69.3( \pm 16.1)$ years old. There were approximately 15 patients from each of the 13 nursing units included in the study.

Active orders were reviewed for the 197 patients, and 197 discrepant orders were identified from a total of 1,424 active orders. The discrepant order rate was 13\% (197/1,424), yielding an average of one discrepant order per patient. Furthermore, $61 \%(120 / 197)$ of patients had at least one discrepancy identified. Figure 2 represents the discrepancies by type. There were a total of 207 discrepancies by type, as there were several orders 
Figure 2. Frequency of medication discrepancies, by type $(n=207)$

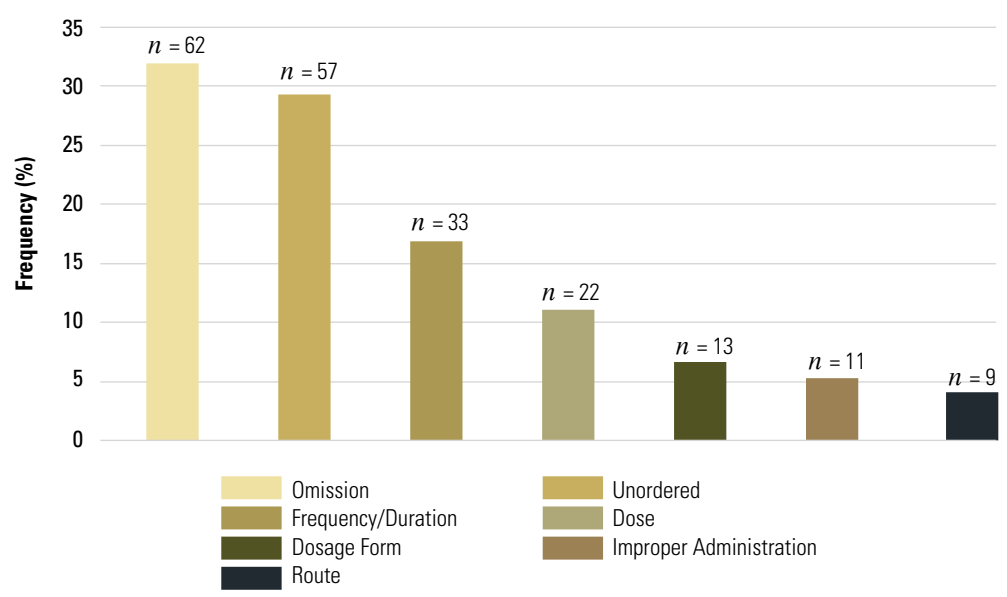

Figure 3. Frequency of medication discrepancies, by documentation source

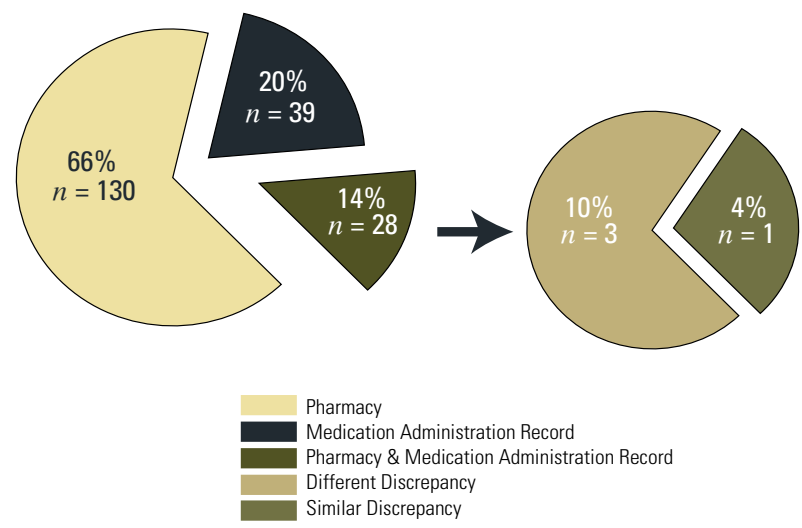

Figure 4. Frequency of assessment of likelihood of error by assessor ( $n=197)$

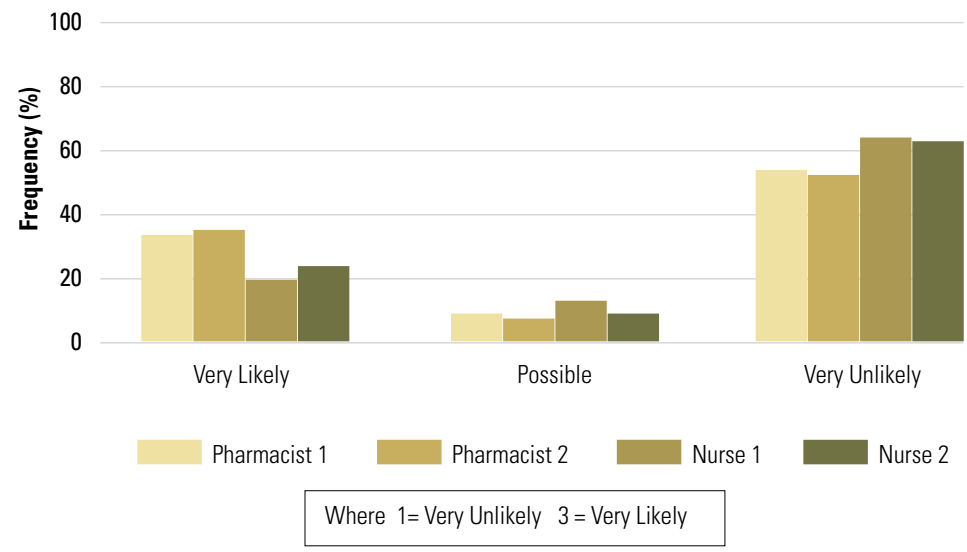

that contained more than one type of discrepancy. The documents in which the discrepancies were identified are shown in Figure 3. The methods of delivery of the medications involved in a discrepancy were (as frequencies): floor stock $54.3 \%$, unit dose $52.7 \%$, multidose format $19.2 \%$, infusions $11.0 \%$, and intravenous admixtures (IVAD) 4.0\%, respectively. (The sum of frequency is greater than $100 \%$, as some items can be obtained via more than one delivery method.)

Age and gender of the patient did not influence the frequency of discrepancy $(\mathrm{p}=0.3469$ and 0.6762 , respectively). Linear regression revealed no statistically significant differences in discrepancy rate by nursing unit $(\mathrm{p}=0.1812)$. There was no difference in the frequency of discrepancies among nursing units, when grouped according to service (i.e., cardiology, orthopedics) $(n=6, p=0.2178)$. Furthermore, correlation coefficients indicated that neither individual nursing unit nor groups of units were predictive of the frequency of discrepancy $(r=$ 0.0823 and 0.0359 , respectively).

Figure 4 represents the judged likelihood of actual error per discrepancy by individual assessor. Assessor agreement was calculated for all pairings using the Cohen kappa coefficient test. For all pairings of assessors, a high level of agreement among them was confirmed through statistical testing (the $\mathrm{Z}$ values for all pairings represent $p$-values $<0.001$, indicating agreement between all pairings of assessors).

Linear regression showed a strong relationship between assessments of likelihood and documentation source of discrepancies $(\mathrm{p}=0)$. Discrepancies were more frequently assessed as likely to lead to error when both the pharmacy profile and MAR were discrepant (average likelihood rating $=2.73)$ (where $1=$ Very Unlikely ... 3=Very Likely), followed by a discrepant MAR (average likelihood rating $=2.36$ ). When all three documents were noted to be discrepant the average likelihood rating fell to 2 , and finally when pharmacy profile was noted to be discrepant the average likelihood ratings were 1.28.

Relationships between assessments of likelihood and discrepancy types were analyzed and showed that discrepancies of "dosage form" were more frequently assessed as "very likely" to have led to an error $(\mathrm{p}=0.0081)$. Discrepancies of 
"dose, route and omissions" were less frequently assessed as "very likely" to have led to an error $(\mathrm{p}=0.0041)$. All other types of discrepancies did not contribute in any way to a "very likely" assessment of likelihood of error.

\section{Discussion}

This study indicates that there are deficiencies in documentation and communication in the MUS in this institution. Fortunately, the majority (87\%) of orders processed within this MUS were processed without discrepancy. Yet, in $13 \%$ of orders - roughly one in eight orders reviewed - there was a discrepancy noted in documentation. These discrepancies represent chances for medication error, as demonstrated in previously published studies (Wilson et al. 1997; Manley et al. 2004).

Discrepancies in documentation in this facility may be explained, in part, by the independent nature of document maintenance by physicians, nurses and pharmacists. The QEII HSC does not currently utilize a joint nursing-pharmacy MAR, nor does it have computerized physician order entry (CPOE). These technologies could lead to improved sharing of medication-related information among MUS users (Ackroyd-Stolarz et al. 2005). An example of a discrepancy that may have been avoided with such technology is an order written by a physician for a topical product to be applied "qd" (an unapproved abbreviation in our facility). The order was subsequently transcribed by nursing as "once daily" and entered by pharmacy as "four times daily." Currently, neither are there processes in place to ensure accuracy of order processing throughout the documentation system (i.e., from ordering to administration).

There were no associations between age, gender, or nursing unit and discrepancy rate. In determining factors most likely to contribute to a discrepancy, the statistical analysis revealed a random spread of error across all the variables studied. This is an important finding, because this indicates that no particular characteristics or areas should be the target for MUS improvement, but rather an overall system enhancement.

The two most common types of discrepancies were omissions $(\approx 31 \%)$ and unordered drugs $(\approx 29 \%)$. The frequency of these discrepancy types suggests that there are system breakdowns occurring at both the transcription and the order entry stage. Specifically, orders written to both initiate and discontinue medications are not being processed through the MUS in the proper manner. This is further supported by the fact that, of the discrepant orders, the pharmacy profile was the documentation source of the discrepancies $66 \%$ of the time. A possible explanation for this high frequency is that orders that do not require pharmacy involvement for drug availability (i.e., floorstocked drugs) are not being sent to the pharmacy for entry on a profile, or the orders are not being processed properly once they reach the pharmacy. Furthermore, more than half of the orders involved in a discrepancy were noted to be floor-stock items.
Although discrepancies were identified, the results from the assessment of the likelihood of actual medication error were promising in that, on average, the assessors felt that these discrepancies would not have led to erroneous drug administration to the patient. In one example the pharmacy profile noted a heparin drip as "active," yet both the physician's order and MAR noted the order as "discontinued." In this scenario, the patient was not likely to still be receiving the heparin despite the discrepancy on the pharmacy profile alone. An interesting result in the assessment was that when the pharmacy profile was found to be the documentation source of a discrepancy, this was deemed "least likely to lead to error" by the assessors. This is an important finding in view of the fact that the majority of discrepancies were noted in the pharmacy profile.

Of greater concern, however, were the discrepancies assessed as "possible" and "very likely." In this study, the assessors deemed discrepancies identified in both the MAR and the pharmacy profile as most likely to lead to actual error. These discrepancies represent an area of priority in implementing process and policy change.

Any discrepancies located at the time of data collection may have been rectified at another point during the hospital stay. Unfortunately, it was only possible to make inferences about the likelihood that a discrepancy led to an actual error, because actual drug administration was not witnessed. The panel members' own professional judgment was the gold standard in this study to determine the likelihood of an actual error occurring. The scale used to document this assessment of likelihood of error has not been validated. Also, it is important to note the bias that exists in the interpretation of the original order. The determination of a discrepancy is based on a pharmacist's own view of what the order reads.

\section{Conclusion}

This study has provided insight into the nature of discrepancies that occur in the documentation used in one facility's MUS. The discrepancies identified suggest that either orders are not reaching pharmacy or orders are not being processed appropriately in pharmacy. Approximately one in eight medication orders were discrepant; however, only a small percentage of these were deemed to have the potential to lead to a medication error. Discrepancies in documentation in this facility may be explained, in part, by the independent nature of document maintenance by physicians, nurses and pharmacists. The location of discrepancies suggests that there are deficiencies in communication between healthcare professionals, so future efforts should be directed toward improving interprofessional communication.

\section{About the Authors}

Jennifer Turple, BSc (Pharm), is a Clinical Pharmacist at the Halifax Infirmary Site of Capital District Health Authority. At the time of writing, she was completing a hospital residency. 
Neil J. MacKinnon, PhD, RPh, is the Associate Director for Research and Associate Professor in the College of Pharmacy at Dalhousie University, and is Associate Professor, School of Health Services Administration and Department of Community Health \& Epidemiology, also at Dalhousie University. He has led several studies on medication safety and is currently editing a book on the safety and quality of the medication use system that will be published in early 2007 .

Bryan Davis, BSc (Pharm), is a pharmacist currently working in the community setting. At the time of writing, he is Director of Unit Dose Services at the Halifax Infirmary Site.

Please direct correspondence to: Neil J. MacKinnon, Associate Director for Research and Associate Professor, School of Health Services Administration and Department of Community Health \& Epidemiology, Dalhousie University, 5968 College Street, Halifax, NS, B3H 3J5; Tel: 902-494-6379. Fax: 902-494-1396. E-mail: neil.mackinnon@dal.ca.

\section{Acknowledgments}

Thanks to Danette Beechinor, Sam Campbell, Brian Gambin, Kathy Hart, Scott Horwood, Kimberley Legere, Bo Lin, Vicki Martin, Tiffany Nguyen and Brian Tuttle.

\section{References}

Ackroyd-Stolarz, S., N. Hartnell and N.J. MacKinnon. 2005. "Approaches to Improving the Safety of the Medication Use System." Healthcare Quarterly 8: 59-64.

Baker, G.R., P.G. Norton, V. Flintoft, R. Blais, A. Brown, J. Cox, E. Etchells, W.A. Ghali, P. Hebert, S.R. Majumdar, M. O’Beirne, L. Palacios-Derflingher, R.J. Reid, S. Sheps and R. Tamblyn. 2004. "The
Canadian Adverse Events Study: The Incidence of Adverse Events among Hospital Patients in Canada." Canadian Medical Association Journal 170(11): 1678-86.

Canadian Council on Health Services Accreditation. 2006. "Patient Safety Goals and ROPs." Retrieved August 17, 2006. <http://www. cchsa-ccass.ca/PatientSafety/psgoalsrops.aspx?culture=en-CA>

Cornish, P.L., S.R. Knowles, R. Marchesano, V. Tam, S. Shadowitz, D.N. Juurlink and E.E. Etchells. 2005. "Unintended Medication Discrepancies at the Time of Hospital Admission." Archives of Internal Medicine 165: 424-29.

Kidney, T. and N.J. MacKinnon. 2001. "Preventable Drug-Related Morbidity and Mortality in Older Adults: A Canadian Cost-of-Illness Model." Geriatrics Today 4: 120.

Kohn, L.T., J.M. Corrigan and M.S. Donaldson (Eds.). 1999. To Err Is Human: Building a Safer Health System. Washington, DC: National Academy Press, Institute of Medicine.

Manley, H.J., D.K. Drayer, M. McClaran, W. Bender and R.S. Muther. 2004. "Drug Record Discrepancies in an Outpatient Electronic Medical Record: Frequency, Type, and Potential Impact on Patient Care at a Hemodialysis Center." Pharmacotherapy 23(2): 231-39.

Nickerson, A., N.J. MacKinnon, N. Roberts and L. Saulnier. 2005. "Drug-Therapy Problems, Inconsistencies and Omissions Identified during a Medication Reconciliation and Seamless Care Service." Healthcare Quarterly 8(Special Issue): 65-72.

Safer Healthcare Now. 2006. Safer Healthcare Now! site. Retrieved August 17, 2006. <http://www.saferhealthcarenow.ca/Default.aspx?fo lderId $=82 \&$ contentId $=124>$

Wilson, A.L., J.J. Jill, R.G. Wilson, K. Nipper and I. Kwon. 1997. "Computerized Medication Administration Records Decrease Medication Occurrences." Pharmacy Practice Management Quarterly 17(1): 17-29.

\section{The best way} to start your day.

Nutrition for the mind. Come to Breakfast with the Chiefs. Join up to 200 of your colleagues for a one-hour discussion on relevant issues in healthcare today. Check out our speakers at www.longwoods.com. By invitation only. For details, contact Lina Lopez at llopez@longwoods.com

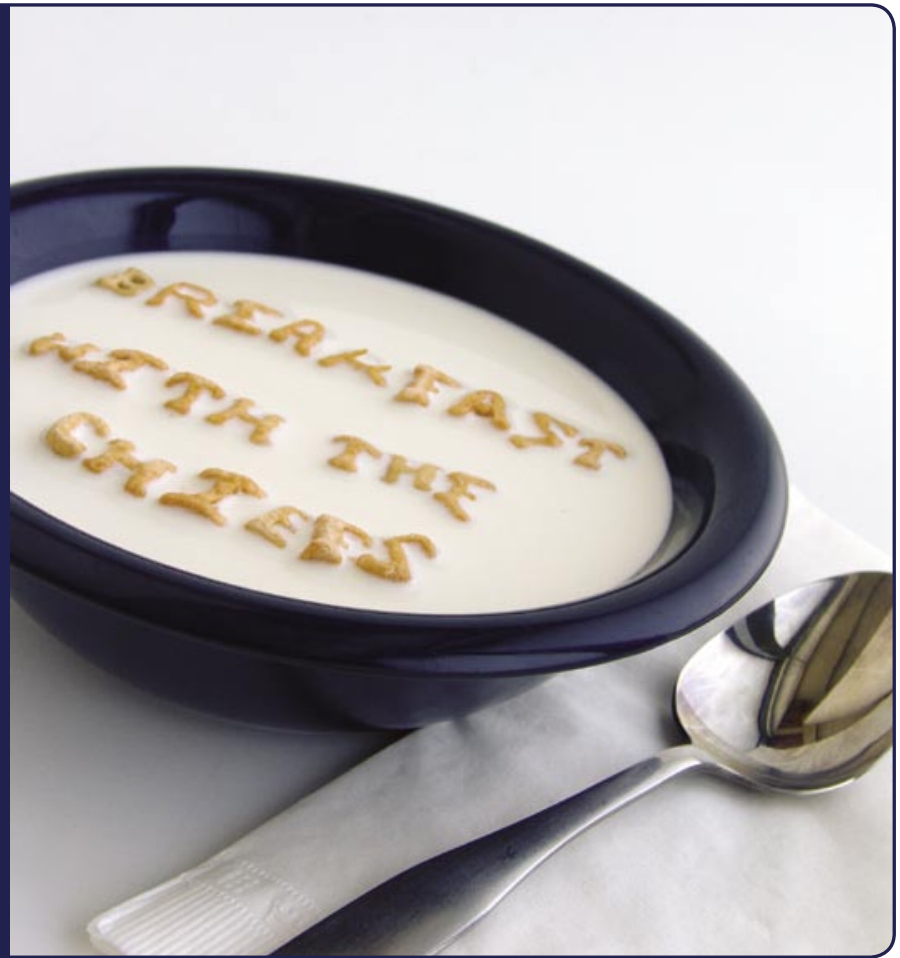

